\title{
Henri Michaux lecteur de Maurice Barrès
}

\author{
David Vrydaghs \\ Université de Liège
}

En 1928, depuis l'Équateur où il séjourne, Henri Michaux fait état auprès de Jean Paulhan de son intérêt pour le premier volume du Culte du moi (1888-1891), trilogie de Maurice Barrès : "Je fais un roman; des vues synthétiques, le contraire du délayage proustien, ne serait pas aux antipodes de Sous l'oeil des Barbares. Vient avec lenteur, sûreté et allure de définitif. Semble devoir s'achever dans quatre ou cinq mois - en quelque sorte classique » (cité par Ouvry-Vial, 1989, p.82). Ce projet n'aboutira pas. Sans doute est-ce pour cela que la critique ne s'est pas interrogée sur les rapports entre ces deux auteurs. On trouve pourtant plusieurs références à l'œuvre de Barrès dans la production de Michaux depuis 1929, date de publication 
d'Ecuador et de Mes propriétés, jusqu'en 1933, quand paraît Un barbare en Asie. De quelle nature sont-elles? Qu'apportent-elles à l'œuvre en cours? Autant de questions jamais posées mais qui méritent assurément de l'être ${ }^{1}$.

Que Michaux ait lu et apprécié Sous l'œil des Barbares au point d'en faire un modèle pour son roman futur n'a par ailleurs rien d'étonnant tant Barrès est un écrivain goûté par la jeune génération dans les années 1920. Raymond Radiguet et Henry de Montherlant lui dédicacent leurs premiers textes (Winock, 1999, p. 183-185). François Mauriac reconnaît que «Barrès a fait beaucoup plus pour [lui] que d'attirer l'attention sur [son] premier livre» (cité par Winock, 1999, p. 185). Louis Aragon, Pierre Drieu La Rochelle et André Malraux peuvent à bon droit être considérés comme les «fils de Barrès » tant leurs œuvres de jeunesse constituent un approfondissement des thèses du Culte du moi et du Roman de l'énergie nationale (1897-1902) voire une réponse à celles-ci (Habrand 2004). Le procès symbolique intenté le 13 mai 1921 par le mouvement dada à l'encontre de l'écrivain est également symptomatique de cet état de chose: l'homme politique y est condamné, non l'écrivain, comme si les futurs surréalistes avaient hésité à jeter le discrédit sur celui qu'ils avaient lu avec passion, ce dont témoignent encore les notes qu'ils lui attribuèrent dans le numéro de Littérature de mars 1921 où il s'agissait de coter diverses personnalités de l'époque : sur une note maximale de 20, Soupault lui donna un 12, Breton un 13, Aragon un 14 et Drieu un 16 (Habrand 2004, p. 26).

\footnotetext{
1 Pour être exact, l'influence de Barrès sur l'écriture de ce livre a déjà été signalée par Didier Alexandre (1995). Nous en faisons cependant une autre lecture, comme on le verra plus loin.
} 
Michaux serait-il dès lors un « fils » de Barrès parmi tant d'autres? Son cas a quand même ceci de particulier qu'à la différence des auteurs cités, il n'a jamais reconnu publiquement sa dette envers Barrès et s'est rapidement défait de son influence. On se demandera par conséquent pourquoi Michaux a privilégié Barrès à ce moment précis de sa carrière pour ensuite l'abandonner. Après 1933 en effet, Michaux est loin de Barrès.

\section{Les territoires du moi : l'héritage barrésien chez Michaux}

Parlant de Barrès à son mentor, Michaux y voyait un modèle pour son roman en cours à deux niveaux au moins : celui des principes de composition; celui du style. En l'absence des brouillons du poète, détruits par celui-ci, il est impossible de vérifier si Michaux a mis sa remarque en pratique. Toujours estil qu'il semble confiner l'importance qu'eut Barrès pour lui à son écriture romanesque; il ne dit mot, à Paulhan, de son journal ni de ses poèmes, auxquels il continue pourtant de travailler.

Une forme d'intertextualité avec Barrès est cependant repérable dans ces textes; mais elle ne consiste pas en reprises stylistiques ni en similitudes au niveau des principes de composition. Qu'a donc emprunté Michaux, si ce n'est le style de Barrès? Sur quoi s'est-il appuyé pour réaliser ces emprunts dans d'autres genres que le roman à thèse?

Dans son étude sur Un barbare en Asie, Didier Alexandre associait la « déconstruction de la logique du savoir, opérée de manière volontiers polémique, à l'intérieur même de la 
connaissance » (p. 208) à la lecture de Barrès par Michaux. L'auteur du Culte du moi mettait en effet en scène l'itinéraire intellectuel d'un jeune Français, Philippe, qui s'était construit une philosophie et un savoir personnels après avoir renié les connaissances et la vision du monde que ses maîtres avaient déposé en lui au cours de son éducation. Le moi de Philippe comprenait ainsi progressivement qu'il devait se défaire des connaissances inculquées par les «barbares » qui l'entouraient s'il voulait se découvrir et s'affirmer².

Le mouvement dialectique identifié par Didier Alexandre se rencontre donc également chez Barrès, mais est-il pour autant imputable à cet auteur? Nous ne le pensons pas. La «déconstruction» critique du savoir constitué - et de la raison - est en effet un topos littéraire dans les années 1920, que l'on rencontre chez Louis Aragon et Georges Bataille, chez Blaise Cendrars et André Breton ou encore à La Nouvelle Revue française, occupée à promouvoir le genre de l'essai contre les savoirs constitués (Macé, 2006, p. 54-74). Les écrivains sont donc nombreux à se défier du savoir constitué et à se comporter comme Anicet ou Philippe avant lui sans être tous des lecteurs de Barrès pour autant ${ }^{3}$.

Un second élément nous incite à nuancer la lecture d'Alexandre. Si Michaux manifeste une mauvaise humeur

\footnotetext{
${ }^{2}$ Chez Barrès, le terme "barbare » ne représente pas une catégorie sociale précise, ce que dit bien ce passage de Sous l'œil des Barbares où Philippe s'exclame: "Qu'on le classe vulgaire ou d'élite, chacun, hors moi, n'est que barbare. À vouloir me comprendre, les plus subtils et bienveillants ne peuvent que tâtonner, dénaturer, ricaner, s'attrister, me déformer enfin, [...]. » (p. 107) 3 On se souviendra que le héros du premier roman d'Aragon était ainsi présenté : «Anicet n'avait retenu de ses études secondaires que la règle des trois unités, la relativité du temps et de l'espace; là se bornaient ses connaissances de l'art et de la vie. » (p. 23)
} 
chronique envers les systèmes de pensée et les professeurs dans la plupart de ses livres, s'il fait dans Ecuador l'éloge du désapprentissage, seule possibilité offerte pour «faire [...] des découvertes » (1998, p. 177) dans un monde gangrené par la raison et la technique, sa position est surtout redevable de son admiration pour les auteurs mystiques, explicite dans son journal de voyage ${ }^{4}$. Pour rappel, ces croyants préféraient à l'orthodoxie la recherche de moyens personnels pour entrer en contact avec le divin (de Certeau, 1982). Barrès n'a donc pu influencer profondément Michaux sur ce point. Sa lecture n'a pu que renforcer ses convictions les plus profondes, non les faire naître.

Michaux a en fait emprunté au Culte du moi ce que celui-ci prétendait offrir : une «culture du moi » (Barrès, 1965, p. 24) : «C'est une culture qui se fait par élaguements et par accroissements : nous avons d'abord à épurer notre moi de toutes les parcelles étrangères que la vie continuellement y introduit, et puis à lui ajouter. Quoi donc? Tout ce qui lui est identique, assimilable.» (p. 30-31) Soit une méthode d'apprentissage, illustrée par l'itinéraire de Philippe dans les romans, qui consiste d'abord à se débarrasser du savoir transmis par les barbares de tous ordres pour retrouver en soi et dans le monde extérieur des éléments sensibles communs

\footnotetext{
4 Cette admiration est d'ailleurs énoncée immédiatement après l'éloge du désapprentissage: "Pour ce qui est de la religion catholique, quand je l'étudiais, je me méfiais beaucoup des évêques, chanoines et professeurs de cours de théologie et de philosophie. Ils sont fort roués, je pensais. Meilleurs me paraissaient le curé d'Ars, blackboulé à tous examens ou questions théologiques, ou saint Joseph de Cupertino surnommé l'âne, et Ruysbroeck l'admirable qui faisait tout de travers, qui ne comprirent point infiniment de détails, mais l'essentiel jusqu'à la moelle : le Dieu qu'il y avait à aimer. » (p. 178)
} 
(Barrès insiste beaucoup sur la nécessité d'accorder ses instincts à ceux d'une race, d'un groupe humain déjà là).

On retrouve ce mouvement dans les premiers écrits de Michaux. Ecuador offre en effet le portrait d'un voyageur dégoûté par ce qu'il voit et entend. Aucun paysage, aucun homme «n'aident à [s]on perfectionnement» (p. 191), souhait explicite du narrateur. Ce premier mouvement, d'élagage, est bientôt contrebalancé par un second mouvement, d'ouverture au monde. Cela n'arrive qu'une fois dans Ecuador, quand le narrateur gagne la forêt tropicale :

Ici, il y a pour moi.

Quand les poètes chantaient les arbres du Nord, je croyais qu'ils le faisaient exprès. Ces arbres nus, sans famille, lisses, abandonnés, hauts troncs, et branches qui n'offrent aucune ouverture (je songe surtout à vous, ô hêtres, que j'ai tant maudits, qu'on voulait me faire admirer, qui portez vers le haut le subit rire malin de toutes vos petites feuilles, qui ne veut rien dire), on ne vous réclame pas, vous tous que j'ai haïs.

Ici, il y a pour moi.

Arbres un peu naïf des tropiques, un peu bête, à grandes feuilles, mes arbres! (p. 169-170)

D’une part donc, l'admiration imposée mais refusée par le narrateur au nom du fait qu'il ne s'y reconnaît pas, soit l'entreprise d'«élaguement» prônée par Barrès face aux discours des barbares; et d'autre part, le choix conscient d'une union avec une partie du monde fondé sur le sentiment de similitude (rendu notamment par «mes arbres», ou "pour moi »), soit l'« accroissement » barrésien.

En Équateur, ce miracle ne se produisit qu'une fois. Le voyageur était plus souvent en butte aux «barbares » de toutes 
sortes qu'au contact d'une réalité qui lui convenait ${ }^{5}$. Dans $M e s$ propriétés, recueil de poèmes en prose écrits en voyage et publiés au retour, la structuration de l'imaginaire barrésien est en revanche continuellement présente, jusqu'à constituer l'ethos des différents narrateurs (Amossy, 1999). Ceux-ci sont tous des poètes qui cherchent dans le monde qui les entoure un «je ne sais quoi de personnel, un je ne sais quoi à [s']adjoindre » (p. 504).

En somme, ils sont comme Philippe autant de moi en quête d'éléments qui leur ressemblent et capables d'accroître leur conscience d'eux-mêmes. Si l'un d'eux est encore "maigre et sec » (p. 470), il possède néanmoins un « terrain » dont il fait état dans "Mes propriétés », texte le plus long du recueil, qui est aussi celui qui lui donne son titre et clôt la première partie du livre. Dans ce véritable précis d'égotisme, le narrateur définit l'espace de son moi à la façon de Barrès. Il pose premièrement qu'aucun enseignement venant de l'extérieur ne peut subsister longtemps en lui :

D'autres fois [...], je vois dans la vie extérieure ou dans un livre illustré, un animal qui me plaît, une aigrette blanche par exemple, et je me dis : Ça, ça ferait bien dans mes propriétés et puis ça pourrait se multiplier, et je prends force notes et je m'informe de tout ce qui constitue la vie de l'animal. Ma documentation devient de plus en plus vaste. Mais quand j'essaie de le transporter dans ma propriété, il lui manque toujours quelques organes essentiels? (p. 465-466)

\footnotetext{
5 Qu'on pense par exemple à cette remarque : " "Indien”, "Indien”, vous voulez me stupéfier avec ça. Un Indien, un homme quoi ! Un homme comme tous les autres, prudent, sans départs, qui n'arrive à rien [...]. / Une fois pour toutes, voici : les hommes qui n'aident pas à mon perfectionnement : zéro. » (p. 191)
} 
C'était déjà ce que constatait Philippe en d'autres termes. Par la suite, le poète compare l'état désolé de son terrain avec la luxuriance des propriétés des autres pour constater que la différence tient à «l'exercice constant de leur intelligence et de leurs capacités extraordinaires » (p. 468). En somme, si son moi ne parvient pas à assimiler des connaissances produites à l'extérieur de lui-même, c'est parce qu'il n'est pas capable d'exercer sa raison ou qu'il ne souhaite pas le faire. Pour autant, le narrateur trouve agrément à ses propriétés, notamment parce qu'elles ne lui « donnent pas cette impression d'absurde qu['il] trouve partout » (p. 467). C'était là encore un sentiment qu'éprouvait Philippe devant le monde extérieur, mais rarement dans son intimité. Le moi du « poète maudit » affirme donc ici sa singularité en même temps que son autarcie. Éloigné du monde commun, il se suffit à lui-même : « Il y a mon terrain et moi; puis il y a l'étranger.» (p. 467) Par l'emploi du terme d'«étranger» pour désigner l'extérieur, Michaux rejoint à nouveau Barrès, pour qui les barbares et leur monde étaient « étrangers » au moi.

Enfin, c'est depuis ce "terrain » éminemment personnel que le poète envisage de poursuivre son œuvre : «Revenons au terrain. Je parlais de désespoir. Non, ça autorise au contraire tous les espoirs, un terrain. Sur un terrain, on peut bâtir, et je bâtirai. Maintenant j'en suis sûr. Je suis sauvé. J'ai une base. » (p. 468) La métaphore foncière, trait ironique décoché à l'encontre de la famille bourgeoise de ce poète, qui lui avait «toujours prédit la plus grande pauvreté et nullité » (p. 469), peut aussi s'interpréter comme une territorialisation dès qu'on prête attention au caractère assertif de chacun des syntagmes (on notera aussi la gradation : « je bâtirai », «j'en suis sûr », « je suis sauvé », «j'ai une base »). Le poète délimite en effet un 
territoire pour sa création. La suite du recueil donne à voir cette «base» sur laquelle Michaux dit s'appuyer: tous les textes retenus dans Mes propriétés constituent de petites rêveries où l'imagination d'un être en souffrance vient l'apaiser un temps par les visions qu'elle lui offre, plus curieuses les unes que les autres. Ainsi, ce «terrain » barrésien devient au fil du recueil une poétique : Michaux fait de ce moi en souffrance la matrice permettant de créer des textes où l'imaginaire domine, où le réel n'est plus qu'un point d'interrogation, où la singularité d'un individu s'affirme pleinement.

On doit maintenant se demander ce qui autorisa Michaux à faire du contenu d'un roman à thèse la structuration d'un «je» lyrique et la «base» d'une poétique. De prime abord, l'écart est en effet considérable entre le genre employé par Barrès pour défendre ses positions et ceux dans lesquels Michaux définit son moi. La distance est toutefois diminuée par le fait que Barrès présentait sa trilogie comme un récit d'apprentissage, puis à la façon d'un «enseignement » (1965, p. 27). Pareillement, Michaux fait d'Ecuador et de Mes propriétés des récits d'apprentissage (Roger, 2005). C'est dans cette communauté de projets que s'établit, au-delà de la diversité générique, une rencontre entre les deux auteurs.

\section{L'Orient se rapproche, Barrès s'éloigne}

Si l'imaginaire de Barrès a influencé Michaux au point de former chez ce dernier une poétique, celui-ci a perdu de son importance à partir du moment où le jeune écrivain a rencontré les philosophies orientales, en Inde et en Chine principalement. 
Un barbare en Asie, qui paraît en 1933 chez Gallimard et relate cette découverte, est ainsi le dernier livre de Michaux où se lit l'attirance de l'auteur pour Barrès, en même temps qu'un fossé apparaît entre leurs conceptions, qui ne cessera de croître par la suite.

En apparence, Michaux reste fidèle à la structuration barrésienne de l'imaginaire :

Ainsi, quand on se retire en soi, et qu'on arrive à supprimer le multiple débat émanent des strates de cette énorme infrastructure [c'est-à-dire un «indébrouillable mélange » d'éléments venant du monde extérieur], on arrive à une paix, à un plan tellement inouï qu'on pourrait se demander si cela aussi ne serait pas le « surnaturel ». (p. 408)

Le moi du voyageur se construit à nouveau par soustraction de tout ce qui lui est nuisible et étranger. Il vient d'ailleurs d'en donner la preuve dans les chapitres qu'il a consacrés aux peuples et cultures asiatiques. Tous ne lui ont pas également plu : les Hindous restent «figés, bétonnés » (p. 285) devant le voyageur, ne lui permettant pas de partager avec eux une forme de connaissance; les portraits du Chinois sont plus enthousiastes, au point qu'au terme de son voyage Michaux se demande : «Est-ce la Chine qui m’a changé? » (p. 386) Toujours est-il qu'à chaque rencontre avec l'Asie, Michaux se demande, comme dans Ecuador et Mes propriétés, «comment prendre » (p. 161).

Michaux ne se rend toutefois pas aux conclusions de la trilogie. Depuis Un homme libre, Barrès reconnaissait que le «moi» ne pouvait prendre sa forme achevée que rapporté à une collectivité dotée d'une tradition millénaire, la France :

[Ce livre décrit] les moments où Philippe se comprit comme un instant d'une chose immortelle. Avec une piété sincère, il 
retrouvait ses origines et entrevoyait ses possibilités futures. À interroger son Moi dans son accord avec des groupes, Philippe en prit le vrai sens. Il l'aperçut comme l'effort de l'instinct pour se réaliser. Il comprit aussi qu'il souffrait de s'agiter, sans tradition dans le passé et tout consacré à cette œuvre viagère. (p. 32-33)

Dès lors, le terme de «barbare» prend un sens supplémentaire. Il désigne encore les défenseurs du kantisme et, plus généralement, du rationalisme et du positivisme; mais il nomme aussi désormais les ennemis de la France, c'est-à-dire pour Barrès les Allemands. L'extension de sens est facilitée par le fait que la philosophie honnie de Barrès est d'origine germanique ${ }^{6}$.

Or Michaux refuse de voir une solution dans le plaidoyer en faveur d'une civilisation, quelle qu'elle soit : elle est toujours « une impasse » (p. 408). Il ajoute encore qu' « un peuple devrait être honteux d'avoir une histoire » (p. 408), signalant ainsi qu'il ne croit pas en l'enracinement d'un moi dans une tradition. L'idée même de collectivité est ensuite raillée :

Il y a eu partout tellement d'invasions de races diverses, Huns , Tartares, Mongols, Normands, etc., et tant d'afflux de religions diverses, néolithique, totémique, solaire, animiste, sumérienne, assyrienne, druidique, romaine, islamique, bouddhique, nestorienne, chrétienne, etc., que personne n'est pur, mais constitue un affreux mélange sans compter les préjugés qu'il

6 Depuis le début des années 1920, le terme «barbare» servait également à qualifier l'Asie. Un débat, qui mêlait écrivains et intellectuels de tous bord, divisait les Français entre partisans d'un renouveau de la pensée occidentale grâce à l'apport des philosophies orientales (Romain Rolland dès 1922 et les surréalistes à partir de 1925 allaient défendre cette idée) et défenseurs de la culture occidentale (Paul Valéry, Henri Massis et Pierre Drieu La Rochelle notamment se rangeraient derrière cette bannière). Les écrivains et intellectuels opposés à la pensée orientale identifiaient alors, grâce au terme de « barbare », l'Asie à l'Allemagne et au bolchévisme (Bandier, 1999, p. 200-201). 
s'est acquis dans sa propre patrie. (p. 408 et p. 1154 pour la fin du passage, supprimée des éditions publiées après 1945).

Dans ce finale, Michaux refuse l'opposition de Barrès entre le moi individuel et les barbares, puis entre le moi collectif et ces derniers. D’après lui, tout individu est nécessairement « barbare », étant donné qu'il est un mélange de civilisations.

C'est d'ailleurs un des sens du titre que Michaux donne à son livre. On peut aussi en voir un autre, qui rend d'autant plus visible le différend entre les deux auteurs : le «barbare » est, depuis l'Antiquité, le représentant d'une civilisation inférieure. En se disant tel, en reconnaissant un extérieur, l'Asie, qui lui est étranger et désirable, Michaux renverse en effet le propos de Barrès. Le moi doit désormais se construire à partir d'idées et de visions du monde qui sont a priori étrangères à sa culture. Pour reprendre les termes de Barrès, l'« accroissement » du moi doit donc se faire au contact de l'étranger (ici l'Asie). Le renversement n'est toutefois pas complet : comme on l'a vu plus haut, Michaux conserve la structuration de l'imaginaire présentée dans Le Culte du moi. Le changement d'un auteur à l'autre porte en fait sur ce qui est reconnu bon pour le moi : chez Barrès, c'est par un accord profond avec la race française que Philippe peut définitivement rejeter les barbares; chez Michaux, c'est par une recherche, entamée dès Ecuador, de ce qui lui ressemble dans d'autres civilisations qu'il peut se grandir.

Le différend entre Michaux et Barrès ne fera ensuite que s'accentuer. Dans la postface de Plume précédé de Lointain intérieur (1938), le poète affirme avec force qu'on ne peut jamais cerner son moi : 
Moi n'est jamais que provisoire (changeant face à un tel, moi $a d$ hominem changeant dans une autre langue, dans un autre art) et gros d'un nouveau personnage [...].

On n'est peut-être pas fait pour un seul moi. On a tort de s'y tenir. Préjugé de l'unité. (Là comme ailleurs la volonté, appauvrissante et sacrificatrice.)

[...]

Il n'est pas un moi. Il n'est pas dix moi. Il n'est pas de moi. Moi n'est qu'une position d'équilibre. (Une entre mille autres continuellement possibles et toujours prêtes.) Une moyenne de «moi », un mouvement de foule. Au nom de beaucoup je signe ce livre. (p. 663)

On voit le chemin parcouru depuis Ecuador : après avoir cherché son moi et trouvé dans le monde de quoi le nourrir et le grandir, Michaux abandonne l'idée même qu'il y en ait un, tant l'entreprise de création lui paraît puiser à différentes sources et se moduler différemment selon la langue pratiquée ou l'art exercé (il peignait régulièrement depuis le début des années 1930). Michaux est loin de Barrès désormais.

\section{Cultiver son moi, c'est créer son territoire}

Il n'empêche que Michaux a été très proche de l'auteur du Culte du moi, au point que son égotisme soit barrésien et que sa poétique en porte la marque de la fin des années 1920 au milieu des années 1930. Comment expliquer cette rencontre et l'utilisation que Michaux fit de Barrès? On pourrait se contenter d'y voir une illustration supplémentaire de ce principe que Michaux rappelait en 1959:

Je ne cherche pas à établir des hiérarchies et je ne m'attarde pas aux écrivains que j'ai aimés. La vie est une nutrition. Il faut sans cesse consommer... et consumer. On aime une pomme, mais on la digère. Un être humain aussi on l'épuise, et une 
œuvre humaine; surtout on dérive petit à petit, appelé par son propre besoin, emporté par son courant à soi. (2004, p. 1463)

Ce passage semble en effet rendre compte de la relation entre Michaux et Barrès: après l'avoir aimé et s'en être profondément inspiré, Michaux l'a «épuisé » et s'est laissé « emporter» ailleurs.

Une autre explication, plus historique, peut également être avancée. Quand on resitue la période barrésienne de Michaux dans son parcours d'écrivain, on constate qu'elle survient à l'heure où le jeune poète commence paradoxalement à se singulariser.

Après des débuts littéraires en Belgique, où il œuvre comme critique culturel (Vrydaghs, 2006), Michaux s'installe à Paris. Il y fréquente des personnalités du monde littéraire : les surréalistes d'abord, dont il adopte un temps les positions (Vrydaghs, 2007); Jules Supervielle et Jean Paulhan ensuite. Ce dernier le fait entrer dans les revues modernistes françaises (Commerce d'abord, la Nouvelle Revue française ensuite) et publie son premier recueil aux éditions Gallimard en 1927. Composé de textes déjà publiés en revues, Qui je fus semble privé de ligne directrice. Des écrits d'inspiration surréaliste ("Principes d'enfant ») y voisinent avec des poèmes qui doivent beaucoup à Apollinaire et aux différents courants modernistes des années 1910 («Adieu à une ville et à une femme»). De telles hésitations coûtent à un jeune poète en temps de crise du secteur poétique. Ce marché connaissait en effet une saturation au début des années 1920, due au surnombre de prétendants par rapport aux places à prendre, le phénomène ne faisant que s'aggraver lorsque les surréalistes prirent position à l'avant- 
garde du champ 7 . Face à cette concurrence, Michaux risquait de passer inaperçu dans ce secteur où "exister c'est différer » (Bourdieu, 1998, p.262), lui qui ne s'affiliait pas à une esthétique reconnue et qui ne proposait pas de poétique novatrice en contrepoint à cette absence d'adhésion.

Avec Ecuador, Mes propriétés et Un barbare en Asie, Michaux affirme progressivement sa manière et sa matière : si ses sources principales sont le mysticisme chrétien et les philosophies asiatiques, il n'est pas un poète spiritualiste de plus, mais d'abord un homme "maigre et sec», à qui rien ne convient, et qui n'a que ses imaginations pour «nourrir» son moi. Un tel ethos le distingue en outre des surréalistes, qui s'affirment sur le mode de la rébellion contre un ordre bourgeois $^{8}$, et de leurs principaux concurrents, comme Blaise Cendrars, confiant dans les pouvoirs du voyage (ce que récuse Michaux dans Ecuador), ou encore les poètes du Grand Jeu, qui s'adressent au divin quand Michaux se centre sur le moi (Bandier 1999, p. 370-373).

Le Culte $d u$ moi participe pleinement de ce positionnement de Michaux dans la littérature de son temps, en ceci qu'il devient la matrice à partir de laquelle le poète se définit et définit sa place ou, si l'on veut, son « terrain ».

\footnotetext{
7 Voir Norbert Bandier, Sociologie du surréalisme, op. cit., p. 36-37.

8 Si Michaux peut à l'occasion se montrer acerbe envers le bourgeois, cela reste circonstanciel chez lui et ne prend jamais la forme d'une critique sociale (à la différence, donc, des surréalistes).
} 


\section{Bibliographie}

ALEXANDRE, Didier. 1995, "Henri Michaux, le barbare ", Revue $d^{\prime}$ histoire littéraire de la France, $9^{\mathrm{e}}$ année, $\mathrm{n}^{\circ} 2$, mars-avril, p. 199-217.

Amossy, Ruth (dir.). 1999, Images de soi dans le discours : la construction de l'ethos, Lausanne, Delachaux \& Niestlé.

ARAGON, Louis. 2001 [1921], Anicet ou le panorama, roman, Paris, Gallimard, coll. « Folio ».

BANDIER, Norbert. 1999, Sociologie du surréalisme, Paris, La Dispute.

BARrÈs, Maurice. 1965, L'CEuvre, tome I, Paris, Au Club de l'Honnête Homme.

Bourdieu, Pierre. 1998, Les Règles de l'art, Paris, Seuil, coll. « Points ».

DE CerteaU, Michel. 1982. La Fable mystique, Paris, Gallimard, coll. « Tel».

Habrand, Tanguy. 2004, Sous l'œil de Barrès: Malraux, Aragon et Drieu La Rochelle dans les années 1920, mémoire de licence, université de Liège.

MACÉ, Marielle. 2006, Le Temps de l'essai : histoire d'un genre en France au XXe siècle, Paris, Belin.

MARTin, Jean-Pierre. 1994, Henri Michaux. Écritures de soi, expatriations, Paris, José Corti.

MichauX, Henri. 1998, OEuvres complètes, tome I, édition de Raymond Bellour, Paris, Gallimard, coll. « Bibliothèque de la Pléiade ». 
MichauX, Henri. 2004, OEuvres complètes, tome III, édition de Raymond Bellour, Paris, Gallimard, coll. «Bibliothèque de la Pléiade ».

OUVRY-VIAL, Brigitte. 1989, Henri Michaux, qui êtes-vous ?, Lyon, La Manufacture.

RoGER, Jérôme. 2005, Ecuador et Un barbare en Asie, Paris, Gallimard, coll. « Foliothèque ».

VRYDAGHS, David. 2005, «Henri Michaux: l'invocation de Lautréamont», dans Paul Aron, Jean-Pierre Bertrand et Pascal Durand (dirs.), La Littérature Maldoror, Tusson, Du Lérot, p. 275-284.

VRYDAGHS, David. 2006, "Henri Michaux au Disque Vert: une participation critique à la littérature », Textyles, n² 29, p. 15-25.

WINOCK, Michel. 1999, Le Siècle des intellectuels, Paris, Seuil, coll. « Points ».

\section{Résumé}

Comme plusieurs jeunes écrivains des années 1920, Henri Michaux (né en 1899) était un lecteur attentif de Maurice Barrès, en particulier de la première trilogie de cet auteur, Le Culte du moi (1888-1891). À la différence de ses contemporains, Michaux n'a jamais reconnu publiquement l'importance de Barrès sur sa formation littéraire et intellectuelle. Pourtant, plusieurs de ses premiers textes (Ecuador, 1929; Mes propriétés, 1929 ; Un barbare en Asie, 1933) sont emplis de références à l'auteur de Sous l'œil des Barbares. Cette intertextualité, ignorée jusqu'ici par la critique spécialisée, fait dans cet article l'objet d'une analyse sociopoétique mettant au jour les effets et les enjeux de cette pratique. 


\begin{abstract}
Like many others young writers of the 20's, Henri Michaux (born in 1899) was a Maurice Barrès's attentive reader. Unlike the contemporary writers, Michaux never admit publicly Barrès's consequence on his literary and intellectual education. However, many Sous l'œil des Barbares's references appear on earl Michaux's texts (Ecuador, 1929 ; Mes propriétés, 1929 ; Un barbare en Asie, 1933). Ignored by the specialized critics, this intertextuality here is studied in the purpose of showing this practice's effects and issues.
\end{abstract}

